\title{
Use of spectrum simulation to optimise collection parameters for accurate and efficient WDS and EDS quantitative analyses
}

Philippe Pinard $^{1}$, Rosie Jones ${ }^{1}$, Simon Burgess ${ }^{2}$ and Peter Statham ${ }^{1}$

${ }^{1}$ Oxford Instruments NanoAnalysis, High Wycombe, United Kingdom, ${ }^{2}$ Oxford Instruments NanoAnalysis, High Wycombe, England, United Kingdom

Besides resolution, sensitivity and speed of acquisition, a key difference between wavelength and energy dispersive spectrometry (WDS/EDS) is the large number of parameters needed to be decided before a WDS acquisition. There is no definitive answer to questions like 'which X-ray line should I use', 'how long should I acquire the peak and backgrounds', 'what background positions should I choose', etc. Beyond rules of thumb and trial and error, Reed and Buckley (1996) and Fournier (2000, 2001) developed tools using stored experimental WD scans or simulation models to assist and optimise the selection of collection parameters [1-3]. Building on these solutions for electron microprobes, we have developed an optimisation engine for combined WDS/EDS analysis in a scanning electron microscope (SEM) to automate the selection of X-ray lines and crystals, background positions and counting times of the WDS acquisition, as well as the EDS collection parameters [4].

The optimisation engine is based on the simulation of a theoretical WD spectrum from first principles. Physical models developed for EDS spectrum simulation [5] are combined with new efficiency, resolution, and peak profile models for WD spectrometers. Models were constructed by extracting the net peak area and full width at half maximum from scans acquired over several X-ray lines and high-order reflections on a representative set of Oxford Instruments Wave spectrometers and crystals. Peak profiles are generated using a Lorentzian function scaled according to the natural width of X-ray transitions and convolved with a Gaussian function based on the spectrometer resolution. Figure 1 shows a comparison between experimental and simulated spectra of pure silver and Ti-6Al-4V alloy.

Spectrum simulation provides critical information for an automated optimisation routine, such as possible interferences between X-ray lines, peak-free background positions, peak/background ratios and absolute $\mathrm{X}$-ray intensities, but requires the accelerating voltage, beam current and sample composition to be known. A short spectrum acquisition by EDS can identify the major and minor elements present in the sample and estimate their concentration with enough accuracy for the purpose of the optimisation. In most SEMs, measuring the beam current requires moving the stage to the Faraday cup location, a cumbersome task when optimising the parameters for an acquisition. A quicker strategy is to rely on the absorbed current, the current measured off the sample. With the approximate sample composition from EDS and analytical expressions for the secondary and backscattered electron yields [6-8], the measured absorbed current can be converted to a beam current. This conversion was tested on different samples at 10, 15 and $20 \mathrm{kV}$ and was found to be accurate within a relative error of $5 \%$. As the accelerating voltage, absorbed current and sample composition vary, the optimisation engine re-simulates a theoretical spectrum and updates its recommended collection parameters. 
For a given element to be measured by WDS, the optimal X-ray line and crystal is determined by considering all possible X-ray lines and eliminating those with an overvoltage less than 1.4 or an interference with X-ray lines of other elements in the sample. The most intense peak is then selected out of the remaining lines. Similarly, the optimal background positions are determined by first checking any interference at the default background positions for the X-ray line. If an interference is found the background position is shifted to the next peak-free region away from the X-ray line. Finding interferences and peak-free regions is straightforward when the full energy range of all crystals is simulated. An example of the X-ray line and background position optimisation is shown in Figure 2 for Pr in a La-rich mineral.

The precision for a quantitative analysis depends on the element concentration, the beam current and the counting times for peak and background measurements. The optimisation therefore consists in adjusting the beam current and counting time to achieve an objective (e.g., the error must be less than 0.05 wt $\%$ ) subject to constraints (e.g., the sample only tolerates currents less than $5 \mathrm{nA}$ ). By either calculating the concentration error for a given peak time or the peak time to achieve a specified error, the other parameter and the absorbed current can be optimised for any acquisition. In all cases, the optimisation engine calculates the counting time at the background positions to yield the smallest error based on the derivation by Scott et al. (1995) and simulated peak-background ratio [9]. Finally, as beam currents needed for WDS are often higher than EDS, especially to measure minor and trace elements, the EDS collection parameters must be adjusted. The optimisation engine finds the appropriate process time to keep the dead time between 30 and $50 \%$ and suitable detector insertion or retraction based on the input count rate.

Setting up a combined WDS/EDS acquisition can be complex even for experts. Using readily available information from the analysis system (EDS spectrum, accelerating voltage and absorbed current), the optimisation engine automatically selects collection parameters to achieve accurate quantitative results in the shortest amount of time.
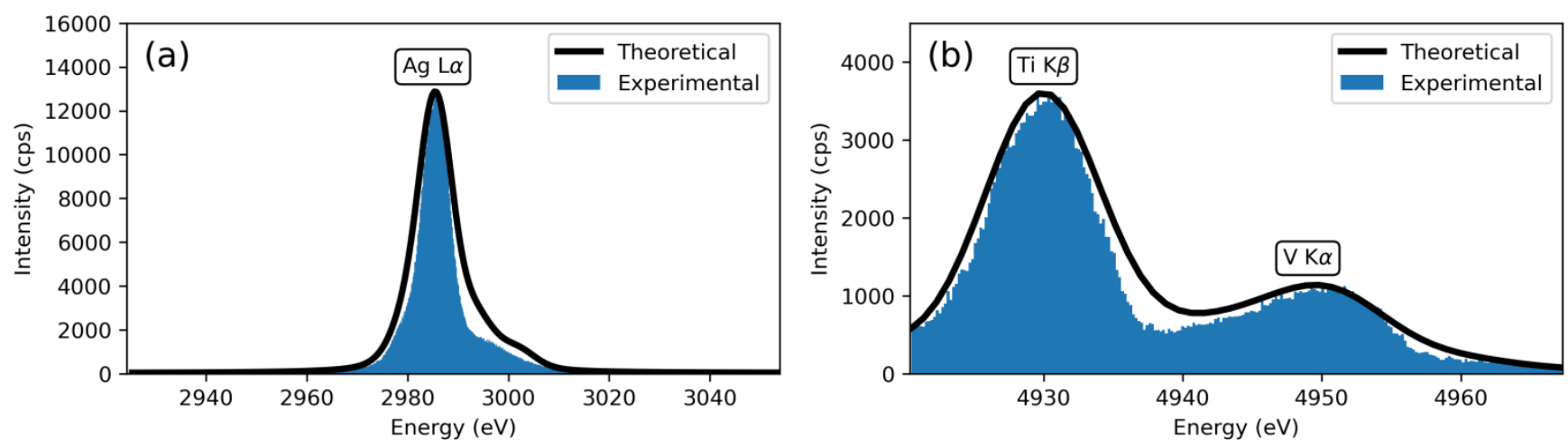

Figure 1. Figure 1. Experimental and theoretical spectra from (a) pure silver on PET crystal and (b) Ti-6AI-4V alloy on LiF crystal. The experimental spectra were acquired at $20 \mathrm{kV}$ on a JEOL 5900 equipped with a Wave spectrometer. 

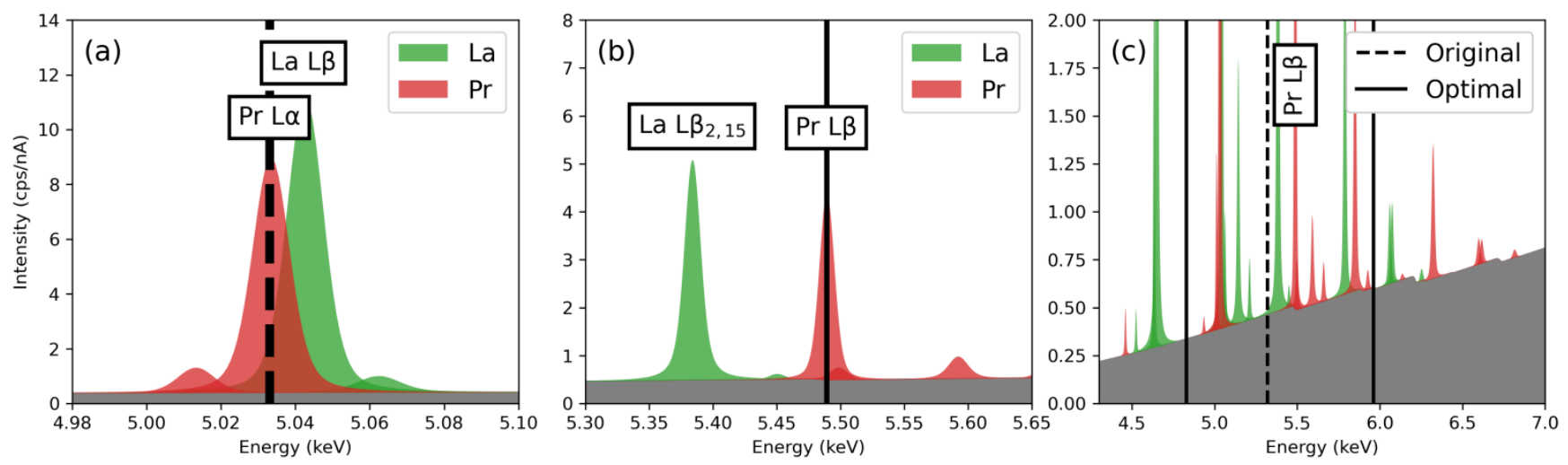

Figure 2. Figure 2. Example of the optimal X-ray line and background positions for $\operatorname{Pr}$ in kosoite, a mineral containing $20 w t \%$ of La. (a) Interference of La L $\beta$ on the Pr L $\alpha$ X-ray line on the LiF crystal, approximately causing a $25 \%$ error on the $\operatorname{Pr} X$-ray intensity. (b) Recommendation to use $\operatorname{Pr} L \beta X$-ray line. (c) Shift of the lower background position of $\operatorname{Pr} L \beta$ due to the interference of $L a L \beta 2,15$.

References

[1] SJB Reed and A Buckley, Mikrochim. Acta 13 (1996), 479.

[2] C Fournier et al., Mikrochim. Acta 132 (2000), 531.

[3] C Fournier et al., Microsc. Microanal. 7 S2 (2001), 674.

[4] S Burgess and PT Pinard, Microsc. Microanal. 26 (S2) (2019), 114.

[5] PJ Statham et al., IOP Conf. Ser.: Mater. Sci. Eng 109 (2016), 012016.

[6] G Love and VD Scott, J. Phys. D 11 (1978), 1369.

[7] Y Lin and DC Joy, Surface and Interface Analysis 37 (2005), 895.

[8] L Reimer, "Scanning Electron Microscopy" (2 $\left.{ }^{\text {nd }}\right)$, Springer (1998).

[9] VD Scott et al., "Quantitative Electron-Probe Microanalysis" (2 $\left.{ }^{\text {nd }}\right)$, Ellis Horwood (1995). 